\title{
I Su Isacco Argiro
}

\section{I.1 Fonti librarie e documentarie}

Nello scolio posto nel margine inferiore del f. 138v degli Harmonica di Tolomeo nel Vat. gr. 176, Argiro afferma di essere stato allievo di Niceforo Gregora: $\delta \iota \omega \rho \theta \omega ́ \sigma \alpha \tau o$

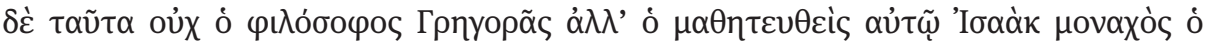
Apyupós. ${ }^{34}$ Oltre a questa attestazione, che consente di legare Argiro a Gregora in un rapporto allievo-maestro e di ancorare così parte della sua attività nel monastero costantinopolitano di Cora, ben poche altre informazioni in merito alla sua vita si ricavano dalle fonti a disposizione. ${ }^{35}$

Nel trattato noto come Computus Paschalis, composto nel 1372 e destinato ad illustrare ad Andronico Ineote il funzionamento dei cicli lunare e solare, Argiro ha inserito una digressione sulla propria infanzia:

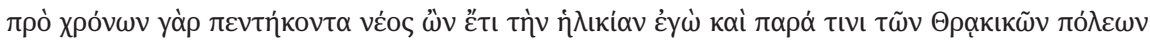

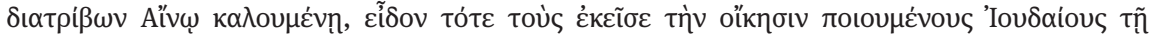

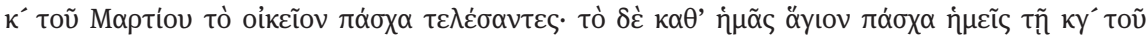

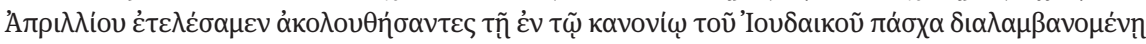

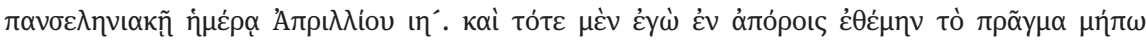

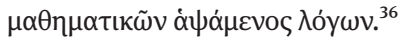

Circa cinquant'anni prima - secondo i calcoli effettuati da Mercati, che ha per primo analizzato il racconto, si farebbe riferimento all'anno 1318 -, Argiro si trovava ad Eno in Tracia. Ancora molto giovane e totalmente digiuno di scienze matematiche, egli era rimasto sorpreso nell'apprendere che tra la Pasqua ebraica, che quell'anno cadeva il 20 marzo, e quella cristiana, fissata per il 23 aprile, potesse intercorrere un intervallo di tempo di più di trenta giorni. Sulla base di questa testimonianza, Mercati ha fissato, a grandi linee, la nascita di Argiro al principio del XIV secolo e quella del suo decesso intorno agli anni '80, vale a dire almeno dieci anni dopo la realizzazione del trattato. ${ }^{37}$

Un'ulteriore informazione si ricava da una delle recensioni del synodikon dell'Ortodossia, nota come $P$, nella quale al nome di Argiro è associato il seguente anatema:

34 Lo scolio al f. 138v è stato riportato anche da Mercati (1931) 229 n. 6, dove tuttavia manca l'indicazione del foglio. Sul Vat. gr. 176 si rimanda infra 132-139.

35 Più in generale si rimanda alla voce su Argiro in PLP nr. 1285.

$36 \mathrm{Nel}$ trattato Argiro affronta, tra le altre, la questione assai controversa della data di Pasqua; su questo trattato si vedano Tihon (1996) 258-259, e Estangüi Gómez (2013) 167-180. Il testo è edito in PG 19, 1276-1316, che riproduce Petau (1630) 359-383; in Baufays (1981), l'edizione si fonda a sua volta sul testo del Marc. gr. Z. 328 (coll. 519), sicuramente una pesante recensione che combina tra l'altro più trattati indipendenti.

37 Mercati (1931) 233-234 e nn. 1-2. 


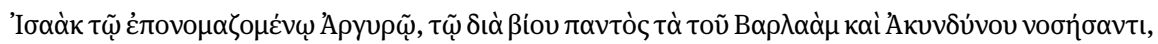

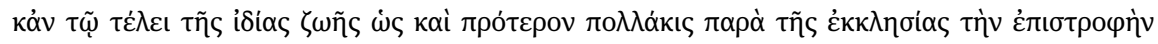

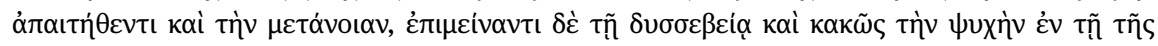

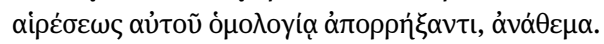

Nonostante le ripetute richieste di abiura e ripensamento da parte della Chiesa, Argiro rimase quindi fedele alle proprie idee e continuò a combattere Palama e i suoi seguaci, come del resto fece lo stesso Gregora, fino alla fine della propria vita. ${ }^{38} \mathrm{La}$ posizione ostile di Argiro nei confronti del palamismo e dei palamiti trova ampia conferma nei quattro trattati teologici a lui attribuiti: De participatione in Deo, De paternitate et filiatione Dei, Contra Dexium, Adversus Cantacuzenum.

Come non si conosce la data precisa della nascita di Argiro, così non vi è certezza sulla data della sua morte. Considerati i termini con cui nel synodikon si fa riferimento ad Argiro, Jean Gouillard, che ne ha curato l'edizione, ha suggerito che egli fosse già morto al momento della redazione della recensione $P$ : dal momento che la composizione di $P$ è stata posta tra il 1383 e il 1391, il 1391 costituisce il terminus ante quem per la sua morte. ${ }^{39}$

Un nuovo elemento può forse anticipare di un decennio circa quest'ultima data. La prima unità dello Scorial. Y.III.21, ff. 1r-115v, raccoglie una serie di scritti matematici e astrologici alla cui copia sono impegnati Argiro e alcuni suoi noti collaboratori. ${ }^{40}$ Una datazione più precisa per la morte di Argiro può desumersi da due annotazioni apposte da Filoteo di Selimbria a margine dei ff. 3rv del codice, dove Argiro ha copiato le Novae Tabulae (ff. 3r-8v). ${ }^{41}$ Nella tavola al f. 3r, Argiro inizia a copiare (ma non completa) una tavola dei cicli vetiquattrennali dei moti medi del sole e della luna, a partire dall'anno $6876=1368$, come è indicato nel titolo:

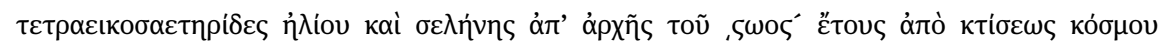

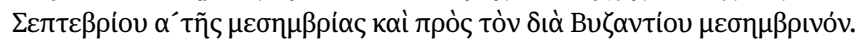

38 Per il synodikon dell'Ortodossia si rinvia a Gouillard (1967), con edizione critica del testo alle pp. 44-118; per questo passo in particolare si veda p. 85, 11. 635-639; ulteriori considerazioni per la data di composizione della redazione $P$ si ritrovano alle pp. 29-34, 239-251. Sulla scomunica ricevuta da Argiro si veda più di recente Estangüi Gómez (2013) 179-180.

39 Gouillard (1967) 239-251.

40 Per questa nuova attribuzione e la descrizione dell'esemplare si veda meglio infra 179.

41 Su Filoteo, allievo di Niceforo Gregora, divenuto in seguito antipalamita e metropolita di Selimbria dal 1362-1389, rinvio a PLP nr. 29896. 
In un'annotazione successiva posta a margine di questa stessa tavola, Filoteo di Selimbria ha precisato che il ciclo in corso include l'anno 6890,1381, ovvero l'anno in cui sta scrivendo:

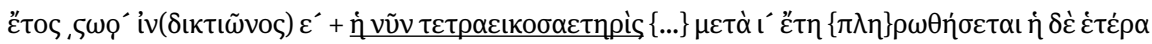

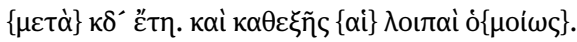

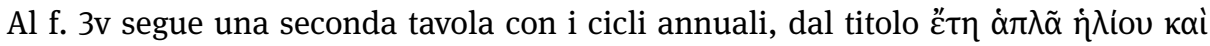
$\sigma \varepsilon \lambda$ ńvns. Nel margine esterno di questo foglio, accanto al quattordicesimo anno del ciclo, Filoteo ha di nuovo riportato l'anno 1381 seguito dall'indizione:

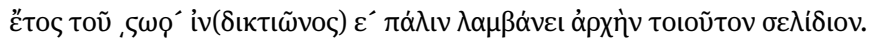

È probabile che queste aggiunte alle tavole siano state inserite da Filoteo in quanto Argiro non era più in grado nel 1381 di aggiornare il computo cominciato anni prima. Sebbene in via del tutto ipotetica, si può supporre che Argiro fosse già morto nel 1381, oppure che il manoscritto non fosse più in suo possesso, motivo per cui Filoteo avrebbe aggiunto tali annotazioni.

Su questa linea del tempo che nasce dai primi decenni del trecento e arriva fino agli anni '80 del secolo emergono le date di composizione di alcune opere. Oltre al Computus Paschalis già ricordato, datato al 1372/3, sono provvisti di datazione i due brevi scritti De novis canonibus e il trattato De usu astrolabii, tutti risalenti all'anno 1367/8. ${ }^{42}$ Intorno al 1369/1370 si data la copia del Tomo Antiocheno antipalamita effettuata da Argiro per Arsenio di Tiro, che oggi si conserva nei tre fogli sciolti Vat. gr. $2335 .{ }^{43}$ Infine, tra le attestazioni librarie in cui resta menzione di Argiro, si dovrà annoverare la sottoscrizione dell'Aristotele Neap. III D 37, sebbene priva di elementi cronologici e topici.

42 Si vedano PLP nr. 1285, Hunger (1978) II, 253, e Acerbi (2016) 187-189. La datazione dell'ultimo trattato si legge in Delatte (1939) 253, righe 11-12.

43 Mercati (1931) 204-205, 209-218; per l'edizione del tomo si rimanda a Polemis (1993) 254-281; per l'attribuzione ad Argiro a Pérez Martín (2008) 448 e, infine, a Estangüi Gómez (2013) 176. Quest'ultimo colloca la copia del codice nel monastero di Cora, diversamente da Pérez Martín (2008). Per la vicinanza con Arsenio di Tiro, metropolita di Antiochia, la studiosa aveva supposto che, dopo la

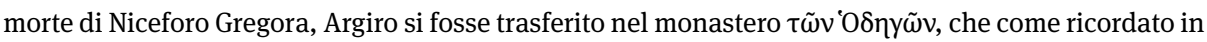
Kresten (2000) 17, fungeva: «als Unterkunft und Aufenthaltsort für Mönche [...] aus dem Patriarchat von Antiocheia , ja für den antiochenischen Patriarchen selbst diente, wenn dieser persönlich in Konstantinopel weilte». 
Sulla base di questi dati non è possibile stabilire se Argiro sia nato a Costantinopoli o in Tracia, ${ }^{44}$ regione di cui parla nel Computus, né se, pratica assai usuale al tempo, egli si sia trasferito nella capitale per completare la propria istruzione, divenendo così allievo di Niceforo Gregora. ${ }^{45} \mathrm{Al}$ rapporto con il milieu costantinopolitano dovranno tuttavia essere ricondotti tanto l'interesse per temi cruciali nel dibattito teologico e dottrinario dell'epoca, come la luce taborica e la processione dello Spirito Santo dal Padre e dal Figlio (Filioque), cui Argiro ha destinato parte della propria produzione letteraria, quanto il suo interesse per l'astronomia, l'astrologia e più in generale la letteratura profana, di cui si colgono cospicue evidenze nella sua produzione letteraria e scrittoria. ${ }^{46}$

\section{I.2 Attribuzioni}

La tabella elenca tutti i manoscritti assegnati ad Argiro. Nella prima colonna sono riportate le segnature degli esemplari, in ordine alfabetico; nella seconda sono specificati i fogli attribuiti ad Argiro e nella terza gli studi in cui le identificazioni sono state proposte. Si dà conto di eventuali discrepanze o specificazioni rispetto all'assegnazione dei fogli avanzate nella bibliografia precedente nelle valutazioni paleografiche seguenti e nelle schede descrittive di ciascun testimone. ${ }^{47}$

\begin{tabular}{lll}
\hline Segnatura & Fogli assegnati ad Argiro & $\begin{array}{l}\text { Studio in cui è formulata } \\
\text { l'attribuzione }\end{array}$ \\
\hline Laur. Plut. 28.13 & $1 \mathrm{r}$-246r & Mondrain (2012) 630 \\
\hline Laur. Plut. 89 sup. 48 & senza indicazione specifica dei fogli & $\begin{array}{l}\text { Bianconi apud Acerbi (2013) } \\
141, \mathrm{n} .50\end{array}$ \\
\hline
\end{tabular}

44 Nella monografia sugli Argiri, Vannier (1975) 15, pone come luogo d'origine della famiglia la Cappadocia.

$45 \mathrm{Si}$ trattava della rotta naturale da percorrere per chi volesse raggiungere e perseguire la carriera degli studi. Molti sono gli esempi che si possono citare al riguardo, come ad esempio Gregorio di Cipro, sulla cui carriera si vedano Constantinides (1982) e Pérez Martín (1996). Su Cora, Teodoro Metochita e Niceforo Gregora, oltre a Ševčenko (1982), si vedano i numerosi lavori destinati ad analizzarne la produzione manoscritta, fra cui Mondrain (2002) 318-322, e (2005), Bianconi (2003) e (2008), cui si rimanda per la bibliografia precedente.

46 Sul ruolo di Argiro nella controversia palamitica si veda ancora Mercati (1931) 233-242. Per quanto concerne i trattati di Argiro inerenti alla controversia, si rinvia qui supra 2 e nn. 9-13. Sull'Adversus Cantacuzenum nello specifico e le miscellanee teologico-dottrinarie di cui Argiro sembra essersi servito per la realizzazione del trattato si vedano infra 110-115; un saggio parziale del modo di lavorare di Argiro si trova in Bianconi (2008) 353-354, e Rigo (2012).

47 Escludo dalla lista i ff. 62v, l. 16-64v; 192r, 1. 16-197v; 226v, 1. 15-229v; 248v del Laur. Plut. 70.5 assegnati ad Argiro da Mondrain (2007) [2008] 168, su cui si veda meglio in seguito, infra 68-69. 
(continued)

\begin{tabular}{|c|c|c|}
\hline Segnatura & Fogli assegnati ad Argiro & $\begin{array}{l}\text { Studio in cui è formulata } \\
\text { l'attribuzione }\end{array}$ \\
\hline Marc. gr. Z. 155 & $195 r-198 r$ & $\begin{array}{l}\text { Bianconi (2008) 365; Mondrain } \\
\text { (2007) [2008] } 168\end{array}$ \\
\hline Marc. gr. Z. 162 & senza indicazione specifica dei fogli & Mondrain (2007) [2008] 168 \\
\hline Marc. gr. Z. 308 & $13 v$ metà-34r & $\begin{array}{l}\text { Bianconi (2008) 357; Mondrain } \\
\text { (2007) [2008] 166-167 }\end{array}$ \\
\hline Marc. gr. Z. 310 & senza indicazione specifica dei fogli & Mondrain (2007) [2008] 166 \\
\hline Marc. gr. Z. 323 & $\begin{array}{l}210 \mathrm{v} ; 215 \mathrm{r}-215 \mathrm{v}, \mathrm{l} .18 ; 216 \mathrm{rv} ; \\
220 \mathrm{r}-221 \mathrm{v}, \mathrm{l} .4 ; 285 \mathrm{r}, \mathrm{l} .14-288 \mathrm{v} \text {, I. } \\
\text { 16; 394r-398v, I. 13; 400r; 468rv; } \\
\text { 471r-476r, l. } 19\end{array}$ & $\begin{array}{l}\text { Bianconi (2008) 358; Mondrain } \\
\text { (2007) [2008] } 166\end{array}$ \\
\hline Neap. III D 37 & $\begin{array}{l}\text { 1r-26v; 28r-37v; 43v-248v, l. 2; } \\
249 r-285 r \text {, l. } 13\end{array}$ & Bianconi (2008) 359-360 \\
\hline $\begin{array}{l}\text { Norimb. Cent. V } \\
\text { App. } 36\end{array}$ & $1 \mathrm{r}-39 \mathrm{v}$ & $\begin{array}{l}\text { Mondrain (2007) [2008] 167; } \\
\text { Murr (1786) 51-52 }\end{array}$ \\
\hline $\begin{array}{l}\text { Norimb. Cent. V } \\
\text { App. } 37\end{array}$ & $1 \mathrm{r}-40 \mathrm{v}$ & $\begin{array}{l}\text { Mondrain (2007) [2008] 167; } \\
\text { Murr (1786) 51-52 }\end{array}$ \\
\hline $\begin{array}{l}\text { Norimb. Cent. V } \\
\text { App. } 38\end{array}$ & $1 \mathrm{r}-49 \mathrm{v}$ & $\begin{array}{l}\text { Düring (1930) xxIv; } \\
\text { Murr (1786) 51-52 }\end{array}$ \\
\hline Par. gr. 940 & $\begin{array}{l}\text { 1-81v, I. } 14 ; 82 \mathrm{v}-83 \mathrm{r}, \text { I. } 25 ; 87 \mathrm{r}-177 \mathrm{v} \text {, } \\
\text { l. } 28\end{array}$ & Mondrain (2007) [2008] 169 \\
\hline Par. gr. 1246 & $161 \mathrm{r}-281 \mathrm{v}, \mathrm{l} .3$ & Polemis (2012) LV \\
\hline Par. gr. 1276 & $132 \mathrm{r}-155 \mathrm{v}, \mathrm{l} .21 ; 156 \mathrm{r}-164 \mathrm{v}$ & $\begin{array}{l}\text { Mondrain (2007) [2008] 168; } \\
\text { Pérez Martín (2008) 447, n. } 221\end{array}$ \\
\hline Par. gr. 1672 & $707 r-869 v$ & qui, $71-72$ \\
\hline Par. gr. 2507 & $1 \mathrm{r}-206 \mathrm{v}$ & Mondrain (2007) [2008] 167 \\
\hline Par. gr. 2758 & $\begin{array}{l}\text { 1r-6v; 8r-14v, I. } 13 ; 16 r v, \text { I. } 26 ; 17 r- \\
38 v, \text { I. } 22 ; 63 r-68 v, \text { I. } 18\end{array}$ & Mondrain (2005) 20 \\
\hline Par. gr. 2821 & $1 \mathrm{r}-11 \mathrm{v} ; 14 \mathrm{r}-103 \mathrm{v}$ & $\begin{array}{l}\text { Bianconi (2008) 361; } \\
\text { Mondrain (2007) [2008] } 169\end{array}$ \\
\hline Parm. 154 & $2 v-114 v$ & $\begin{array}{l}\text { Bianconi (2008) 361; Mondrain } \\
\text { (2007) [2008] } 169\end{array}$ \\
\hline Prah. XXV.C.31 & $1 \mathrm{r}-11 \mathrm{v}$ & Mondrain (2007) [2008] 169 \\
\hline Scorial. Y.III.21 & $3 r-8 v ; 10 r-88 v$ & qui, 7 e 179 \\
\hline Vat. Pal. gr. 174 & $55 ; 121 \mathrm{r}-302 \mathrm{v}$ & Menchelli (2014) 184 \\
\hline Vat. gr. 81 & $292 v-293 r$ & Bianconi (2008) 359 \\
\hline Vat. gr. 176 & $11 \mathrm{v}$, l. 29-27r; $100 \mathrm{v}-192 \mathrm{v}$ & Düring (1930) XxXIII \\
\hline
\end{tabular}


(continued)

\begin{tabular}{|c|c|c|}
\hline Segnatura & Fogli assegnati ad Argiro & $\begin{array}{l}\text { Studio in cui è formulata } \\
\text { l'attribuzione }\end{array}$ \\
\hline Vat. gr. 573 & annotazioni a $11 \mathrm{r} ; 14 \mathrm{r}$ & qui, 137 \\
\hline Vat. gr. 604 & annotazioni a 44v; 46rv-47r & Mercati (1931) 241 \\
\hline Vat. gr. 678 & annotazioni a 86v; $103 \mathrm{v}$ & Bianconi (2008) 362 \\
\hline Vat. gr. 1094 & $\begin{array}{l}\text { 110r-111r; 115v, I. 23-116r; 166v, I. } \\
\text { 16-167r, 205-227; e annotazioni a } \\
\text { 194v-195rv; 201v, I. 20-202r }\end{array}$ & Polemis (2012) Lv \\
\hline Vat. gr. 1096 & $65 r-148 ; 94 r-110 r$ & Mercati (1931) 241 \\
\hline Vat. gr. 1102 & $\begin{array}{l}\text { 1r-9r, I.18; 15r, I. 15-23v; 25v, Il. } \\
\text { 26-29; 31r, I. 14-34r, I. 14; 35r, I. } \\
\text { 7-45v, I. } 9\end{array}$ & $\begin{array}{l}\text { Mercati (1931) 271-273, } \\
359-403\end{array}$ \\
\hline Vat. gr. 1115 & $1 \mathrm{r}-58 \mathrm{r} ; 93 \mathrm{r}-114 \mathrm{v} ; 117 \mathrm{r}-156 \mathrm{r}$ & Mercati (1931) 165-166 \\
\hline Vat. gr. 1721 & $1 \mathrm{v}, 1.2-8 \mathrm{v} ; 10 \mathrm{r}-15 \mathrm{v} ; 17 \mathrm{r}-26 \mathrm{v} ; 28 \mathrm{r}-32 \mathrm{v}$ & Bianconi (2008) 364 \\
\hline Vat. gr. 2335 & Ir, Iv, II. 1-23, IIrv, IIIrv & Mercati (1931) 240-241 \\
\hline
\end{tabular}

